\section{SAT0263 PREDICTORS OF REMISSION MAINTENANCE AND SUCCESSFUL THERAPY DISCONTINUATION IN PATIENTS WITH NON-RADIOGRAPHIC AXIAL SPONDYLOARTHRITIS (NR-AXSPA) WHO ACHIEVED SUSTAINED REMISSION ON OPEN-LABEL ADALIMUMAB (ADA) TREATMENT}

J. Sieper $^{1}$, R. Landewé ${ }^{2}$, M. Magrey ${ }^{3}$, J. Anderson ${ }^{4}$, S. Zhong ${ }^{4}$, X. Wang ${ }^{4}$, A. Lertratanakul ${ }^{4}{ }^{1}$ Charité Universitätsmedizin Berlin, Berlin, Germany; ${ }^{2}$ University of Amsterdam, Amsterdam, Netherlands; ${ }^{3}$ Case Western Reserve University School of Medicine at MetroHealth Medical Center, Cleaveland; ${ }^{4}$ AbbVie Inc. North Chicago, USA

Background: Sustained remission is an important treatment goal in patients (pts) with non-radiographic axial SpA (nr-axSpA). Factors predicting successful remission maintenance are unknown.

Objectives: We sought to identify predictors of remission maintenance in $\mathrm{nr}-$ axSpA pts who achieved remission after open-label (OL) adalimumab (ADA) treatment in the ABILITY-3 trial (NCT01808118) and were subsequently randomised to continuation or withdrawal of ADA therapy.

Methods: ABILITY-3 enrolled adult pts with nr-axSpA with objective evidence of active MRI inflammation in the SI joints or spine or elevated high-sensitivity CRP at screening, active disease at baseline (ASDAS $\geq 2.1, B A S D A l \geq 4$, and Patient's Assessment of Total Back Pain score $\geq 4$ ), and inadequate response to $>2$ NSAIDs (table 1). Pts received ADA $40 \mathrm{mg}$ every other wk during a 28-wk OL lead-in period. Pts who achieved sustained remission, defined as ASDAS inactive disease [ID] score $<1.3$ at wks $16,20,24$, and 28 , were randomised to doubleblind withdrawal (placebo; PBO) or continued ADA for 40 wks during period 2 (study wk 68). Stepwise logistic regression was used to identify predictors of sustained remission in those in the continued ADA and withdrawal (PBO) groups. Remission maintenance in period 2 was assessed with the following: ASAS partial remission (PR; score $\leq 2.0$ ) and ASDAS ID at wk 68, ASAS PR and ASDAS ID at every visit, and ASDAS ID for $\geq 5$ of 10 visits.

Abstract SAT0263 - Table 1. Characteristics at Baseline and Wk 28

\begin{tabular}{|c|c|c|c|c|c|c|c|}
\hline \multicolumn{8}{|c|}{ Table. Characteristics at Baseline a } \\
\hline $\begin{array}{l}\text { Variable } \\
\text { Baseline, mean (SD) }\end{array}$ & $\begin{array}{c}\text { ADA } \\
(40 \mathrm{mg} \\
\text { EOW) } \\
\mathrm{n}=152\end{array}$ & $\begin{array}{c}\text { PBO } \\
\mathrm{n}=153\end{array}$ & $P$ Value & Wk 28, mean (SD) & $\begin{array}{c}\mathrm{ADA} \\
\left(\begin{array}{c}\mathrm{ADO} \\
\mathrm{mg} \\
\mathrm{EOW} \\
\mathrm{n}=152\end{array}\right. \\
\mathrm{n}=15\end{array}$ & $\begin{array}{c}\text { PBO } \\
n=153\end{array}$ & $P$ Value \\
\hline Age, y & $\frac{1-1.2}{34.7(10.3)}$ & $35.3(10.2)$ & 0.611 & ASDAS & $0.7(0.4)$ & $0.6(0.4)$ & 0.355 \\
\hline $\begin{array}{l}\text { Female, n (\%) } \\
\text { SpA symptom }\end{array}$ & $\begin{array}{l}56(37) \\
6.4(6.9)\end{array}$ & $\begin{array}{l}60(39) \\
7.1(6.8)\end{array}$ & $\begin{array}{l}0.724 \\
0.358\end{array}$ & $\begin{array}{l}\text { BASDAI } \\
\text { BASFI }\end{array}$ & $\begin{array}{l}0.8(0.8) \\
0.7(1.0)\end{array}$ & $\begin{array}{l}0.7(0.7) \\
0.7(1.2)\end{array}$ & 0.145 \\
\hline duration, y & & & & & & & \\
\hline $\begin{array}{l}\text { HLA-B27 positive, } \mathrm{n} \\
(\%)\end{array}$ & $132(87)$ & $134(88)$ & 0.866 & Hs-CRP, mg/L & $1.5(2.4)$ & $1.4(1.8)$ & 0.647 \\
\hline $\begin{array}{l}\text { ASDAS } \\
\text { BASDAI }\end{array}$ & $\frac{3.5(0.9)}{6.8(14)}$ & $\frac{3.5(0.8)}{6.8(15)}$ & 0.851 & $\begin{array}{l}\text { Pt total back pain } \\
\text { PGA }\end{array}$ & $\frac{1.1(1.4)}{0.8(10)}$ & $\frac{1.0(1.4)}{0.9(1)}$ & 0.567 \\
\hline BASFI & $5.1(2.3)$ & $5.0(2.3)$ & 0.776 & $\begin{array}{l}\text { SPARCC SI joint } \\
\text { scoret }\end{array}$ & $2.6(6.1)$ & $2.5(4.0)$ & 0.775 \\
\hline Hs-CRP, mg/L & $9.9(14.1)$ & $9.1(13.3)$ & 0.576 & $\begin{array}{l}\text { SPARCC spine } \\
\text { score: }\end{array}$ & $1.2(3.8)$ & $1.2(3.8)$ & 0.889 \\
\hline Prt total back pain & $7.0(1.7)$ & $7.0(1.8)$ & 0.946 & & & & \\
\hline & $6.6(1.5)$ & $6.4(1.4)$ & 0.150 & & & & \\
\hline $\begin{array}{l}\text { SPARCC SI joint } \\
\text { score }\end{array}$ & $8.5(12.8)$ & $10.3(13.4)$ & 0.226 & & & & \\
\hline $\begin{array}{l}\text { SPARCC spine score } \\
\text { SPA }\end{array}$ & $3.3(7.5)$ & $3.6(7.2)$ & 0.671 & & & & \\
\hline 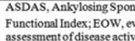 & & & Bathent & $\begin{array}{l}\text { Spondylitis Disease Ac } \\
\text { hs-CRP, high-sensitiv }\end{array}$ & 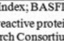 & $\begin{array}{l}\text { Bath Ankylos } \\
\text { POA, physic }\end{array}$ & $\begin{array}{l}\text { Spondylitis } \\
\text { iglobal }\end{array}$ \\
\hline & & & & & & & \\
\hline
\end{tabular}

Results: By wk 68, 100/145 (69\%) ADA pts had not flared, 41\% achieved ASAS PR and $56 \%$ ASDAS ID at wk $68 ; 23 \%$ achieved ASAS PR and $29 \%$ ASDAS ID at every visit, while $70 \%$ achieved ASDAS ID for $\geq 5$ of 10 visits. By wk $68,70 / 148$ (47\%) PBO pts had not flared, $28 \%$ achieved ASAS PR and $33 \%$ ASDAS ID at wk $68 ; 14 \%$ achieved ASAS PR and $15 \%$ ASDAS ID at every visit, while $52 \%$ achieved ASDAS ID for $\geq 5$ of 10 visits. Shorter symptom duration, lower wk 28 BASDAl, and male sex were associated with absence of flare in the continued ADA group. Lower wk 28 ASDAS was associated with absence of flare with ADA withdrawal. Lower wk 28 ASDAS consistently predicted ASAS PR and ASDAS ID at wk 68 , ASAS PR and ASDAS ID at every visit, and ASDAS ID for $\geq 5$ of 10 visits in pts who continued or withdrew ADA.

Conclusions: In nr-axSpA pts who achieved remission after 28-wk OL ADA therapy, lower wk 28 ASDAS is a consistent predictor of remission maintenance using all definitions in both the adalimumab continuation and withdrawal groups, except absence of flare in the adalimumab continuation group, suggesting early aggressive treatment may be beneficial in achieving sustained remission.

Acknowledgements: AbbVie funded the study, contributed to its design and participated in data collection, analysis and interpretation of the data, and in writing, review, and approval of the publication. Medical writing support was provided by Maria Hovenden, PhD, and Janet E. Matsuura, PhD, of Complete Publication Solutions, LLC (North Wales, PA) and was funded by AbbVie.

Disclosure of Interest: J. Sieper Consultant for: AbbVie, Janssen, Lilly, Merck, Novartis, Pfizer, Sun Pharma, and UCB, Speakers bureau: AbbVie, Janssen, Lilly, Merck, Novartis, and Pfizer., R. Landewé Grant/research support from: Abbott, Amgen, Centocor, Novartis, Pfizer, Roche, Schering-Plough, UCB, and Wyeth, Consultant for: consulting or advisory board fees from Abbott/AbbVie, Ablynx, Amgen, Astra-Zeneca, Bristol Myers Squibb, Celgene, Janssen (formerly Centocor), Galapagos, GlaxoSmithKline, Novartis, Novo-Nordisk, Merck, Pfizer Roche, Schering-Plough, TiGenix, UCB, and Wyeth, Employee of: he is director of Rheumatology Consultancy BV, a registered Dutch company., Speakers bureau: Abbott/AbbVie, Amgen, Bristol Myers Squibb, Janssen (formerly Centocor), Merck, Pfizer, Roche, Schering-Plough, UCB, Wyeth, M. Magrey Grant/ research support from: Amgen, AbbVie, and UCB Pharma, Consultant for: UCB and Janssen, J. Anderson Shareholder of: AbbVie, Employee of: AbbVie, S. Zhong Shareholder of: AbbVie, Employee of: AbbVie, X. Wang Shareholder of: AbbVie, Employee of: AbbVie, A. Lertratanakul Shareholder of: AbbVie, Employee of: AbbVie

DOI: 10.1136/annrheumdis-2018-eular.2632

\section{SAT0264 DOES BIOLOGICAL DISEASE-MODIFYING ANTI- RHEUMATIC DRUG NAÏVE VERSUS NON-NAÏVE PATIENTS WITH INFLAMMATORY JOINT DISEASES HAVE SIMILAR GOLIMUMAB DRUG SURVIVAL AND EFFICACY? DATA FROM THE PROSPECTIVE OBSERVATIONAL NOR-DMARD STUDY}

B. Michelsen ${ }^{1,2}$, J. Sexton ${ }^{1}$, T.K. Kvien' ${ }^{1}{ }^{1}$ Dept. of Rheumatology, Diakonhjemmet Hospital, Vinderen, Oslo; ${ }^{2}$ Hospital of Southern Norway Trust, Kristiansand, Norway

Background: Knowledge is limited regarding the impact of previously used biological disease modifying anti-rheumatic drugs (bDMARD) on golimumab drug survival and efficacy in inflammatory joint diseases (IJD).

Objectives: To explore golimumab drug survival and efficacy in bDMARD naïve vs. non-naïve IJD patients, as well as predictors of golimumab discontinuation. Methods: From the observational prospective multicenter Norwegian-DMARD (NOR-DMARD) study rheumatoid arthritis (RA), psoriatic arthritis (PsA) and axial spondyloarthritis (ax-SpA) patients starting golimumab were included. Drug survival was explored by Kaplan-Meier analyses with comparison of bDMARD naïve vs. non-naïve patients with log rank test, stratified by diagnosis. 3 month responses were compared with independent $t$-test, and with ANCOVA adjusted for age, gender, disease duration and baseline values of the respective composite scores. Univariable and multivariable Cox regression analyses including age gender, disease duration, smoking, concomitant synthetic DMARDs (sDMARDs) and previous bDMARD use were performed to identify predictors of golimumab discontinuation.

Results: Mean (SD) age of the $163 \mathrm{RA}, 267$ PsA and 382 ax-SpA patients was $51.2(14.1) / 48.1(11.7) / 41.8(11.1)$ years, disease duration $11.3(10.0) / 8.7(9.0) /$ 10.6 (11.2) years,\%females $76.1 / 55.1 / 44.1 \%$, respectively. Golimumab drug

\begin{tabular}{|c|c|c|c|c|c|c|c|}
\hline \multirow[t]{4}{*}{ Diagnosis } & \multirow{4}{*}{$\begin{array}{l}\text { Composite } \\
\text { score }\end{array}$} & \multicolumn{5}{|c|}{ Changes (baseline minus 3 months) } & \multirow{4}{*}{ p-value } \\
\hline & & \multirow{3}{*}{$\begin{array}{l}\text { Unadjusted analyses } \\
\text { Mean (SD) } \\
\text { bDMARD naïve } \\
\text { patients }\end{array}$} & & \multirow[t]{3}{*}{ p-value } & \multirow{2}{*}{\multicolumn{2}{|c|}{$\begin{array}{l}\text { Adjusted analyses } \\
\text { Estimated marginal means }(95 \% \mathrm{Cl})\end{array}$}} & \\
\hline & & & & & & & \\
\hline & & & $\begin{array}{c}\text { Previous bDMARD } \\
\text { users }\end{array}$ & & $\begin{array}{l}\text { bDMARD naïve } \\
\text { patients }\end{array}$ & $\begin{array}{c}\text { Previous bDMARD } \\
\text { users }\end{array}$ & \\
\hline \multirow[t]{2}{*}{ RA } & $\Delta$ DAS28 & $-1.1(1.3)$ & $-0.7(3.42)$ & 0.39 & $-1.7(-2.6,-0.7)$ & $-0.6(-1.5,0.2)$ & 0.08 \\
\hline & $\Delta \mathrm{CDAl}$ & $-9.0(10.8)$ & $-9.0(13.2)$ & 0.99 & $-11.7(-14.5,-8.9)$ & $-8.7(-11.2,-6.1)$ & 0.10 \\
\hline \multirow[t]{3}{*}{ PsA } & $\Delta$ DAS28 & $-1.2(1.1)$ & $-0.5(1.0)$ & $<0.001$ & $-1.0(-1.3,-0.8)$ & $-0.6(-0.8,-0.3)$ & 0.01 \\
\hline & $\Delta$ CDAl & $-7.1(7.8)$ & $3.8(7.5)$ & 0.005 & $-6.2(-7.6,-4.8)$ & $-4.8(-6.3,-3.3)$ & 0.18 \\
\hline & $\triangle$ BASDAI & $-1.4(2.1)$ & $-0.6(2.1)$ & 0.08 & $-1.2(-1.6,-0.8)$ & $-0.9(-1.4,-0.5)$ & 0.38 \\
\hline \multirow[t]{2}{*}{ Ax-SpA } & $\triangle$ BASDAI & $-1.9(2.4)$ & $-0.9(2.1)$ & $<0.001$ & $-1.9(-2.3,-1.6)$ & $-0.8(-1.2,-0.4)$ & $<0.001$ \\
\hline & $\triangle$ ASDAS & $-1.2(1.3)$ & $-0.5(1.1)$ & $<0.001$ & $-1.1(-1.3,-0.9)$ & $-0.5(-0.8,-0.3)$ & $<0.001$ \\
\hline
\end{tabular}

Identified predictors for golimumab discontinuation were [Hazard Ratio (95\% Cl)]: RA, None; PsA, female gender (1.63 (1.18-2.25), p=0.003) and no concomitant sDMARDs (1.39 (1.01-1.90),

$\mathrm{p}=0.04)$; ax-SpA, female gender [1.97 $(1.50-2.60), \mathrm{p}<0.001]$. 